\title{
Article \\ The Solutions of Initial (-Boundary) Value Problems for Sharma-Tasso-Olver Equation
}

\author{
Lingxiao $\mathrm{Li}^{1, * \mathbb{C}}$, Mingliang Wang ${ }^{1,2}$ and Jinliang Zhang ${ }^{1}$ \\ 1 School of Mathematics \& Statistics, Henan University of Science \& Technology, Luoyang 471000, China; \\ mlwang@haust.edu.cn (M.W.); 9901914@haust.edu.cn (J.Z.) \\ 2 School of Mathematics \& Statistics, Lanzhou University, Lanzhou 730000, China \\ * Correspondence: lilingxiao@haust.edu.cn
}

check for updates

Citation: Li, L.; Wang, M.; Zhang, J. The Solutions of Initial (-Boundary) Value Problems for Sharma-TassoOlver Equation. Mathematics 2022, 10, 441. https://doi.org/10.3390/ math10030441

Academic Editor:

Christopher Goodrich

Received: 8 January 2022

Accepted: 28 January 2022

Published: 29 January 2022

Publisher's Note: MDPI stays neutral with regard to jurisdictional claims in published maps and institutional affiliations.

Copyright: () 2022 by the authors Licensee MDPI, Basel, Switzerland. This article is an open access article distributed under the terms and conditions of the Creative Commons Attribution (CC BY) license (https:// creativecommons.org/licenses/by/ $4.0 /)$.
Abstract: A nonlinear transformation from the solution of linear KdV equation to the solution of Sharma-Tasso-Olver (STO) equation is derived out by using simplified homogeneous balance (SHB) method. According to the nonlinear transformation derived here, the exact explicit solution of initial (-boundary) value problem for STO equation can be constructed in terms of the solution of initial (-boundary) value problem for the linear $\mathrm{KdV}$ equation. The exact solution of the latter problem is obtained by using Fourier transformation.

Keywords: STO equation; nonlinear transformation; SHB method; solution of initial (-boundary) value problem; linear KdV equation; Fourier transformation; an even (odd) extension technique

\section{Introduction}

In recent years, many researchers [1-11] (and refs. therein) pay more attention to the Sharma-Tasso-Olver (STO) equation due to its appearance in scientific applications. STO equation reads,

$$
u_{t}+3 \alpha u^{2} u_{x}+3 \alpha\left(u u_{x}\right)_{x}+\alpha u_{x x x}=0
$$

Equation (1) appears as an evolution equation that possesses an infinitely many symmetries [12], and can be regarded as a member of the hierarchy of higher order Burgers Equation [13] in the form,

$$
u_{t}+\left[\sum_{n=0}^{N} \alpha_{n}\left(\frac{\partial}{\partial x}+u\right)^{n} u\right]_{x}=0
$$

where $N$ is a positive integer.

In fact, $N=2, \alpha_{0}=\alpha_{1}=0, \alpha_{2}=\alpha$, the higher order Burgers equation becomes STO Equation (1).

In the present paper, first of all, we consider the initial value problem of STO equation as follows,

$$
\begin{gathered}
u_{t}+3 \alpha u^{2} u_{x}+3 \alpha\left(u u_{x}\right)_{x}+\alpha u_{x x x}=0, t>0,-\infty<x<\infty \\
u(x, 0)=f(x),-\infty<x<\infty
\end{gathered}
$$

Second, we consider the initial-boundary value problem of STO equation in the form,

$$
\begin{gathered}
u_{t}+3 \alpha u^{2} u_{x}+3 \alpha\left(u u_{x}\right)_{x}+\alpha u_{x x x}=0, t>0, x>0 \\
u(0, t)=0, t \geq 0, u(x, 0)=f(x), x \geq 0
\end{gathered}
$$

Third, we consider the initial-boundary value problem of STO equation with nonlinear boundary condition as follows,

$$
u_{t}+3 \alpha u^{2} u_{x}+3 \alpha\left(u u_{x}\right)_{x}+\alpha u_{x x x}=0, t>0, x>0
$$




$$
\left(u_{x x}+3 u u_{x}+u^{3}\right)_{x=0}=\mu(t), u(x, 0)=f(x), x \geq 0
$$

The boundary condition in (6) means the flux is given at $x=0, f(x)$ and $\mu(t)$ are given continuous functions. To the first problem, we will find a function $u=u(x, t)$ such that $u=u(x, t)$ satisfies STO Equation (1) in the domain $-\infty<x<\infty, t>0$, and when $t$ goes to zero, $u(x, t)$ approaches to $f(x)$, namely $u(x, t)$ satisfies the initial condition (2); To the second problem, we will find a function $u=u(x, t)$ such that $u=u(x, t)$ satisfies STO Equation (3) in the domain $0<x<\infty, t>0$, and when $t$ goes to zero, $u(x, t)$ approaches to $f(x), x \geq 0$, and when $x$ goes to zero, $u(x, t)$ approaches to $u(0, t)=0, t \geq 0$. To the third problem, we will find a function $u(x, t)$ such that $u=u(x, t)$ satisfies STO Equation (5) in the domain $0<x<\infty, t>0$, and when $t$ goes to zero, $u(x, t)$ approaches to $f(x)$, $x \geq 0$ and when $x$ goes to zero, the boundary condition in (6) is satisfied.

The paper is organized as follows: In Section 2, using simplified homogeneous balance (SHB) method, we will derive out a nonlinear transformation from the solution of a linear equation to the solution of STO equation. In Sections 3-5, based on the nonlinear transformation derived in Section 2, the solutions of the first problem, the second problem and the third problem are constructed in terms of the solutions of corresponding linear problems, respectively. In Section 6, some conclusions are made.

\section{Derivation of Nonlinear Transformation}

The STO Equation (1) can be rewritten as the conservation law form,

$$
\frac{\partial u}{\partial t}+\alpha \frac{\partial}{\partial x}\left(u_{x x}+3 u u_{x}+u^{3}\right)=0
$$

Considering homogeneous balance between $u_{x x}$ and $u^{3}$ (or $\left.u u_{x}\right)(m+2=3 m$, or $m+2=2 m+1$, both equations imply $m=1$ ), according to the SHB method [14-17], we suppose that the solution of Equation (1) is of the form,

$$
u(x, t)=A \frac{\varphi_{x}}{\varphi}
$$

where the constant $A$ and function $\varphi=\varphi(x, t)$ are to be determined later. From (7) it is easy to derive out that,

$$
\left\{\begin{array}{l}
u_{t}=A\left(\frac{\varphi_{x t}}{\varphi}-\frac{\varphi_{t} \varphi_{x}}{\varphi^{2}}\right), u_{x x}=A\left(\frac{\varphi_{x x x}}{\varphi}-\frac{3 \varphi_{x} \varphi_{x x}}{\varphi^{2}}+2 \frac{\varphi_{x}^{3}}{\varphi^{3}}\right), \\
u u_{x}=A^{2}\left(\frac{\varphi_{x} \varphi_{x x}}{\varphi^{2}}-\frac{\varphi_{x}^{3}}{\varphi^{3}}\right), u^{3}=A^{3} \frac{\varphi_{x}^{3}}{\varphi^{3}}
\end{array}\right.
$$

Substituting (8) into (1), yields,

$$
\begin{aligned}
& \frac{\partial u}{\partial t}+\alpha \frac{\partial}{\partial x}\left(u_{x x}+3 u u_{x}+u^{3}\right)=A \frac{\varphi_{x t}}{\varphi}-A \frac{\varphi_{x} \varphi_{t}}{\varphi^{2}}+\alpha A \frac{\partial}{\partial x}\left(\frac{\varphi_{x x x}}{\varphi}\right) \\
& +\alpha \frac{\partial}{\partial x}\left[\left(3 A^{2}-3 A\right) \frac{\varphi_{x} \varphi_{x x}}{\varphi^{2}}\right]+\alpha \frac{\partial}{\partial x}\left[\left(A^{3}-3 A^{2}+2 A\right) \frac{\varphi_{x}^{3}}{\varphi^{3}}\right]=0 .
\end{aligned}
$$

Setting the coefficient of $\frac{\varphi_{x} \varphi_{x x}}{\varphi^{2}}$ and $\frac{\varphi_{x}^{3}}{\varphi^{3}}$ in expression (9) to zero, yields,

$$
A^{3}-3 A^{2}+2 A=0 \text { and } 3 A^{2}-3 A=0 \rightarrow A=1
$$

Using the result (10), the expression (7) becomes,

$$
u(x, t)=\frac{\varphi_{x}}{\varphi}
$$

And the expression (9) can be simplified as,

$$
\frac{\partial u}{\partial t}+\alpha \frac{\partial}{\partial x}\left(u_{x x}+3 u u_{x}+u^{3}\right)=\frac{1}{\varphi}\left(\frac{\partial}{\partial x}-\frac{\varphi_{x}}{\varphi}\right)\left(\varphi_{t}+\alpha \varphi_{x x x}\right)=0
$$


Provided that $\varphi=\varphi(x, t)$ satisfies the linear $\mathrm{KdV}$ equation,

$$
\varphi_{t}+\alpha \varphi_{x x x}=0
$$

From (11)-(13), we come to the conclusion that if $\varphi=\varphi(x, t)$ is a solution of linear $\mathrm{KdV}$ Equation (13), substituting it into the expression (11), we have the solution of STO Equation (1). Thus, the expression (11) and the linear KdV Equation (13) together comprises a nonlinear transformation from the solution of linear KdV Equation (13) to the solution of STO Equation (1).

\section{The Solution of Initial Value Problem for STO Equation}

Based on the nonlinear transformation derived in Section 2, we will solve initial value problem in the following,

$$
\begin{gathered}
\frac{\partial u}{\partial t}+\alpha \frac{\partial}{\partial x}\left(u_{x x}+3 u u_{x}+u^{3}\right)=0, t>0,-\infty<x<\infty \\
u(x, 0)=f(x),-\infty<x<\infty
\end{gathered}
$$
by (11),

According to the nonlinear transformation, the solution of STO equation (1) is given

$$
u(x, t)=\frac{\varphi_{x}}{\varphi}
$$

where $\varphi=\varphi(x, t)$ is the solution of Equation (13). When $t$ goes to zero, from (11) and initial condition (2), yields,

$$
f(x)=u(x, 0)=\frac{\varphi_{x}(x, 0)}{\varphi(x, 0)}
$$

Which implies that,

$$
\varphi(x, 0)=\exp \left[\int_{-\infty}^{x} f(\xi) \mathrm{d} \xi\right]
$$

Thus, we acquire the initial value problem of linear Equation (13) with initial condition (14) as follows,

$$
\begin{gathered}
\varphi_{t}+\alpha \varphi_{x x x}=0, t>0,-\infty<x<+\infty \\
\varphi(x, 0)=\exp \left[\int_{-\infty}^{x} f(\xi) \mathrm{d} \xi\right],-\infty<x<\infty
\end{gathered}
$$

We will prove that if $\varphi=\varphi(x, t)$ solves initial value problems (13) and (14) for linear $\mathrm{KdV}$ equation, then expression (11) solves the initial value problems (1) and (2) for STO equation.

In fact, the solution of initial value problems (13) and (14) is given by,

$$
\varphi(x, t)=\int_{-\infty}^{\infty} E(x-\xi, t) \exp \left[\int_{-\infty}^{\xi} f(\eta) \mathrm{d} \eta\right] \mathrm{d} \xi
$$

where $E(x, t)$ is the solution of the initial value problem in the following,

$$
\begin{gathered}
E_{t}+\alpha E_{x x x}=0, t>0,-\infty<x<\infty \\
E(x, 0)=\delta(x),-\infty<x<\infty
\end{gathered}
$$

where $\delta(x)$ is $\delta$ function. $E(x, t)$ is called the fundamental solution of initial value problems (13) and (14), and $E(x, t)$ can be obtained by using Fourier transformation [13], as follows,

$$
E(x, t)=\frac{1}{(3 \alpha t)^{\frac{1}{3}}} A_{i}\left[\frac{x}{(3 \alpha t)^{\frac{1}{3}}}\right]
$$


where,

$$
A_{i}[y]=\frac{1}{\pi} \int_{0}^{\infty} \cos \left(y s+\frac{1}{3} s^{3}\right) \mathrm{d} s
$$

which is called Airy function [18]. When the variable $t$ goes to zero, $E(x, t)$ approaches to the $\delta$ function,

$$
E(x, 0)=\delta(x)
$$

Substituting (18) into (15), we have the solution of initial value problems (13) and (14) as,

$$
\varphi(x, t)=\frac{1}{\pi(3 \alpha t)^{\frac{1}{3}}} \int_{-\infty}^{\infty}\left[\int_{0}^{\infty} \cos \left(\frac{x-\xi}{(3 \alpha t)^{\frac{1}{3}}} s+\frac{1}{3} s^{3}\right) \mathrm{d} s\right] \exp \left[\int_{-\infty}^{\xi} f(\eta) \mathrm{d} \eta\right] \mathrm{d} \xi
$$

and when $t$ goes to zero, (20) approaches to (see ref. [13]),

$$
\varphi(x, 0)=\int_{-\infty}^{\infty} \delta(x-\xi) \exp \left[\int_{-\infty}^{\xi} f(\eta) \mathrm{d} \eta\right] \mathrm{d} \xi=\exp \left[\int_{-\infty}^{x} f(\eta) \mathrm{d} \eta\right] .
$$

Substituting (20) into (11), canceling the factor $\frac{1}{\pi(3 \alpha t)^{\frac{1}{3}}}$, we have the solution of initial value problems (1) and (2) for STO equation, as follows,

$$
u(x, t)=\frac{\frac{\partial}{\partial x} \int_{-\infty}^{\infty}\left[\int_{0}^{\infty} \cos \left(\frac{x-\xi}{(3 \alpha t)^{\frac{1}{3}}} s+\frac{1}{3} s^{3}\right) \mathrm{d} s\right] \exp \left[\int_{-\infty}^{\xi} f(\eta) \mathrm{d} \eta\right] \mathrm{d} \xi}{\int_{-\infty}^{\infty}\left[\int_{0}^{\infty} \cos \left(\frac{x-\xi}{(3 \alpha t)^{\frac{1}{3}}} s+\frac{1}{3} s^{3}\right) \mathrm{d} s\right] \exp \left[\int_{-\infty}^{\xi} f(\eta) \mathrm{d} \eta\right] \mathrm{d} \xi}
$$

When $t$ goes to zero, using (21) and (22), yields,

$$
u(x, 0)=f(x),-\infty<x<\infty
$$

The initial condition (2) is satisfied.

\section{The Solution of Initial-Boundary Value Problem for STO Equation}

The initial-boundary value problems (3) and (4) can be rewritten as,

$$
\begin{gathered}
\frac{\partial u}{\partial t}+\alpha \frac{\partial}{\partial x}\left(u_{x x}+3 u u_{x}+u^{3}\right)=0, x>0, t>0 \\
u(0, t)=0, t \geq 0 ; u(x, 0)=f(x), x \geq 0
\end{gathered}
$$

Similarly, based on the nonlinear transformation expressed by (11) with (13), and using the boundary condition and initial condition (4), we can acquire the corresponding initial-boundary value problem of linear $\mathrm{KdV}$ equation as follows,

$$
\begin{aligned}
& \varphi_{t}+\alpha \varphi_{x x x}=0, t>0,0<x<\infty \\
& \varphi_{x}(0, t)=0, t \geq 0 ; \\
& \varphi(x, 0)=\exp \left[\int_{0}^{x} f(\eta) \mathrm{d} \eta\right], 0 \leq x<\infty .
\end{aligned}
$$

It is obvious that if $\varphi=\varphi(x, t)$ solves initial-boundary value problems (23) and (24) for linear equation, then expression (11) solves the initial-boundary value problems (3) and (4) for STO equation.

Now using an even extension technique, we solve the initial-boundary problems (23) and (24).

Consider the following initial value problem,

$$
\Phi_{t}+\alpha \Phi_{x x x}=0, t>0,-\infty<x<\infty
$$




$$
\Phi(x, 0)=\Phi_{0}(x),-\infty<x<+\infty,
$$

where $\Phi_{0}(x)$ is an even function: $\Phi_{0}(x)=\Phi_{0}(-x)$, which is defined by,

$$
\Phi_{0}(x)=\exp \left[\int_{0}^{x} f(\eta) \mathrm{d} \eta\right], x>0 \Phi_{0}(x)=\exp \left[\int_{0}^{-x} f(\eta) \mathrm{d} \eta\right], x<0
$$

According to the discussion that from (13) to (21) in Section 3, the solution of initial value problems (25) and (26) is given by,

$$
\Phi(x, t)=\int_{-\infty}^{\infty} E(x-\xi, t) \Phi_{0}(\xi) \mathrm{d} \xi
$$

where $E(x, t)$ is expressed by (18).

Since $\Phi_{0}(x)$ is an even function, $\frac{\partial}{\partial x} \Phi_{0}=0$. if $x=0$, So, when $x>0$, the function (27) satisfies the initial-boundary value problems (23) and (24), thus the solution of which can be written as,

$$
\begin{aligned}
& \varphi(x, t)=\Phi(x, t)_{x>0}=\int_{-\infty}^{\infty} E(x-\xi, t) \Phi_{0}(\xi) \mathrm{d} \xi \\
& =\int_{-\infty}^{0} E(x-\xi, t) \exp \left[\int_{0}^{-\xi} f(\eta) \mathrm{d} \eta\right] \mathrm{d} \xi+\int_{0}^{\infty} E(x-\xi, t) \exp \left[\int_{0}^{\xi} f(\eta) \mathrm{d} \eta\right] \mathrm{d} \xi \\
& =\int_{0}^{\infty}[E(x-\xi, t)+E(x+\xi, t)] \exp \left[\int_{0}^{\xi} f(\eta) \mathrm{d} \eta\right] \mathrm{d} \xi
\end{aligned}
$$

Substituting (28) into (11) we have the solution of initial-boundary value problems (3) and (4) for STO equation, as follows,

$$
u(x, t)=\frac{\frac{\partial}{\partial x} \int_{0}^{\infty}[E(x-\xi, t)+E(x+\xi, t)] \exp \left[\int_{0}^{\xi} f(\eta) \mathrm{d} \eta\right] \mathrm{d} \xi}{\int_{0}^{\infty}[E(x-\xi, t)+E(x+\xi, t)] \exp \left[\int_{0}^{\xi} f(\eta) \mathrm{d} \eta\right] \mathrm{d} \xi}
$$

where $E(x, t)$ is expressed by (18).

\section{Initial-Boundary Value Problem for STO Equation with Nonlinear Boundary Condition}

The initial-boundary value problems (5) and (6) can be rewritten as,

$$
\begin{gathered}
\frac{\partial u}{\partial t}+\alpha \frac{\partial}{\partial x}\left(u_{x x}+3 u u_{x}+u^{3}\right)=0, x>0, t>0 \\
\left(u_{x x}+3 u u_{x}+u^{3}\right)_{x=0}=\mu(t), u(x, 0)=f(x), x \geq 0
\end{gathered}
$$

Using the nonlinear transformations expressed in Equations (11) and (13), the initialboundary value problems (5) and (6) can be changed into the initial-boundary value problem for linear $\mathrm{KdV}$ equation as,

$$
\begin{aligned}
& \varphi_{t}+\alpha \varphi_{x x x}=0, t>0,0<x<\infty \\
& \varphi(0, t)=\exp \left[-\alpha \int_{0}^{t} \mu(\eta) \mathrm{d} \eta\right], t \geq 0 \\
& \varphi(x, 0)=\exp \left[\int_{0}^{x} f(\eta) \mathrm{d} \eta\right], x \geq 0
\end{aligned}
$$

We can prove that if $\varphi=\varphi(x, t)$ solves initial-boundary value problems (30)-(32) for linear $\mathrm{KdV}$ equation, then expression (11) solves the initial-boundary value problems (5) and (6) for STO equation. 
In fact, it is obvious that the expression (11) satisfies Equation (5) and initial condition (6). We only examine the boundary condition (6) is satisfied. Substituting (11) into the left hand side of the boundary condition (when $x>0$ ) in (6), and using Equation (6), yields,

$$
u_{x x}+3 u u_{x}+u^{3}=\left(\frac{\varphi_{x}}{\varphi}\right)_{x x}+3\left(\frac{\varphi_{x}}{\varphi}\right)\left(\frac{\varphi_{x}}{\varphi}\right)_{x}+\left(\frac{\varphi_{x}}{\varphi}\right)^{3}=\frac{\varphi_{x x x}}{\varphi}=\frac{-\frac{1}{\alpha} \varphi_{t}}{\varphi}
$$

When $x$ goes to zero, using boundary condition (31), then (33) becomes,

$$
\left(u_{x x}+3 u u_{x}+u^{3}\right)_{x=0}=\mu(t)
$$

This completes the proof.

Based on the superposition principle of a linear problem, the solution of initialboundary value problems (30)-(32) for linear KdV equation is given by,

$$
\varphi(x, t)=\varphi^{(1)}(x, t)+\varphi^{(2)}(x, t)
$$

where $\varphi^{(1)}(x, t)$ satisfies the initial-boundary value problem as,

$$
\begin{gathered}
\varphi_{t}^{(1)}+\alpha \varphi_{x x x}^{(1)}=0, t>0,0<x<\infty \\
\varphi^{(1)}(0, t)=0, t \geq 0 \\
\varphi^{(1)}(x, 0)=\exp \left[\int_{0}^{x} f(\eta) \mathrm{d} \eta\right], x \geq 0,
\end{gathered}
$$

and $\varphi^{(2)}(x, t)$ satisfies the initial-boundary value problem as,

$$
\begin{gathered}
\varphi_{t}^{(2)}+\alpha \varphi_{x x x}^{(2)}=0, t>0,0<x<\infty \\
\varphi^{(2)}(0, t)=\exp \left[-\alpha \int_{0}^{t} \mu(\eta) \mathrm{d} \eta\right], t \geq 0 \\
\varphi^{(2)}(x, 0)=0, x \geq 0 .
\end{gathered}
$$

Now using an odd extension technique, we solve the initial-boundary problems (35)-(37).

Consider the following initial value problem,

$$
\begin{gathered}
\Phi_{t}+\alpha \Phi_{x x x}=0, t>0,-\infty<x<\infty \\
\Phi(x, 0)=\Phi_{0}(x),
\end{gathered}
$$

where $\Phi_{0}(x)$ is an odd function: $\Phi_{0}(x)=-\Phi_{0}(-x)$, which is defined by,

$$
\begin{aligned}
& \Phi_{0}(x)=\exp \left[\int_{0}^{x} f(\eta) \mathrm{d} \eta\right], x>0 ; \\
& \Phi_{0}(x)=-\exp \left[\int_{0}^{-x} f(\eta) \mathrm{d} \eta\right], x<0
\end{aligned}
$$

According to the discussion that from (13) to (21) in Section 3, the solution of initial value problems (41) and (42) is given by,

$$
\Phi(x, t)=\int_{-\infty}^{\infty} E(x-\xi, t) \Phi_{0}(\xi) \mathrm{d} \xi
$$

where $E(x, t)$ is expressed by (18). 
Since $\Phi_{0}(x)$ is an odd function, $\Phi_{0}=0$, if $x=0$. So, when $x>0$, the function (43) satisfies the initial-boundary value problems (35)-(37), thus the solution of which can be written as,

$$
\begin{aligned}
& \varphi^{(1)}(x, t)=\Phi(x, t)_{x>0}=\int_{-\infty}^{\infty} E(x-\xi, t) \Phi_{0}(\xi) \mathrm{d} \xi \\
& =-\int_{-\infty}^{0} E(x-\xi, t) \exp \left[\int_{0}^{-\xi} f(\eta) \mathrm{d} \eta\right] \mathrm{d} \xi+\int_{0}^{\infty} E(x-\xi, t) \exp \left[\int_{0}^{\xi} f(\eta) \mathrm{d} \eta\right] \mathrm{d} \xi \\
& =\int_{0}^{\infty}[E(x-\xi, t)-E(x+\xi, t)] \exp \left[\int_{0}^{\xi} f(\eta) \mathrm{d} \eta\right] \mathrm{d} \xi
\end{aligned}
$$

where $E(x, t)$ is expressed by (18).

Using Duhamel principle [13,19], the solution of problems (38)-(40) is given by,

$$
\varphi^{(2)}=\frac{\partial}{\partial t} \int_{0}^{t} K(x, t-\tau) \exp \left[-\alpha \int_{0}^{\tau} \mu(\eta) \mathrm{d} \eta\right] \mathrm{d} \tau
$$

where,

$$
K(x, t)=1-\int_{0}^{\infty}[E(x-\xi, t)-E(x+\xi, t)] \mathrm{d} \xi
$$

which is the solution of the following problem,

$$
K_{t}+\alpha K_{x x x}=0, t>0,0<x<\infty K(0, t)=1, t \geq 0, K(x, 0)=0, x \geq 0 .
$$

Substituting (44) and (45) into (34) we have the solution of problems (30)-(32) as,

$$
\begin{aligned}
& \varphi=\int_{0}^{\infty}[E(x-\xi, t)-E(x+\xi, t)] \exp \left[\int_{0}^{\tau} f(\eta) \mathrm{d} \eta\right] \mathrm{d} \xi \\
& +\frac{\partial}{\partial t} \int_{0}^{t} K(x, t-\tau) \exp \left[-\alpha \int_{0}^{\tau} \mu(\eta) \mathrm{d} \eta\right] \mathrm{d} \tau .
\end{aligned}
$$

Substituting (46) into (11), we have the solution of the problems (5) and (6).

\section{Conclusions}

In this paper, solving the initial (-boundary) value problem of STO equation has been changed into solving the initial (-boundary) value problem of the linear KdV equation based on the nonlinear transformation which has been derived by using SHB method for STO equation. The initial (boundary) condition in the latter problem is derived from the given initial (boundary) condition in the former one. Using Fourier transformation, we have obtained the solution of initial value problem for the linear KdV equation. Using an even (odd) extension technique, we have obtained the solution of initial-boundary value problem for the linear $\mathrm{KdV}$ equation.

Author Contributions: Investigation, J.Z.; methodology, M.W.; writing—original draft preparation, L.L. All authors have read and agreed to the published version of the manuscript.

Funding: This research was funded by the National Natural Science Foundation of China, grant number 12101193.

Institutional Review Board Statement: Not applicable.

Informed Consent Statement: Not applicable.

Data Availability Statement: Not applicable.

Conflicts of Interest: The authors declare no conflict of interest.

\section{References}

1. Chen, A. Multi-kink solutions and soliton fission and fusion of the Sharma-Tasso-Olver equation. Phys. Lett. A 2010, 734, 2340-2345. [CrossRef]

2. Wazwaz, A.M. New solitons and kinks solutions to the Sharma-Tasso-Olver equation. Appl. Math. Comput. 2007, 188, 1205-1213. [CrossRef] 
3. Erbas, B.; Yusufoglu, E. Exp-function method for constructing exact solutions of Sharma-Tasso-Olver equation. Chaos Solitons Fractals 2009, 41, 2326-2330. [CrossRef]

4. Lian, Z.; Luo, S.Y. Symmetries and exact solutions of the Sharma-Tasso-Olver equation. Nonlinear Anal. 2005, 63, e1167-e1177. [CrossRef]

5. Wang, S.; Tang, X.Y.; Luo, S.Y. Soliton fission and fusion, Burgers equation and Sharma-Tasso-Olver equation. Chaos Solitons Fractals 2004, 21, 231-239. [CrossRef]

6. Zayed, E.M.E. A note on the modified simple equation method to Sharma-Tasso-Olver equation. Appl. Math. Comput. 2011, 216, 3962-3964. [CrossRef]

7. Shang, Y.; Qin, J.; Huang, Y. Abundant exact and explicit solitary wave and periodic wave to the Sharma-Tasso-Olver equation. Appl. Math. Comput. 2008, 202, 532-538. [CrossRef]

8. Shang, Y.; Huang, Y.; Yuan, W. Backlund transformations and abundant exact explicit solutions of the Sharma-Tasso-Olver equation. Appl. Math. Comput. 2011, 217, 7172-7183. [CrossRef]

9. Pan, J.T.; Chen, W.Z. A new auxiliary equation method and its application to the Sharma-Tasso-Olver equation. Phys. Lett. A 2009, 373, 3118-3121. [CrossRef]

10. Zhou, Y.; Yang, F.; Liu, Q. Reduction of the Sharma-Tasso-Olver equation, and series solutions. Commun. Nonlinear Sci. Numer. Simul. 2011, 16, 641-646. [CrossRef]

11. Wang, C. Dynamics behavior of traveling waves for the Sharma-Tasso-Olver equation. Nonlinear Dyn. 2016, 85, 1-8. [CrossRef]

12. Olver, P.J. Evolution equations possessing infinitely many symmetries. J. Math. Phys. 1977, 18, 1212-1215. [CrossRef]

13. Wang, M.L. Higher Burgers Equation. Acta Math. Sci. 1986, 6, 353-360. [CrossRef]

14. Wang, M.L.; Li, X.Z. Simplified homogeneous balance method and its applications to the Whitham-Broer-Kaup model equations. J. Appl. Math. Phys. 2014, 2, 823-827. [CrossRef]

15. Wang, M.L.; Zhang, J.L.; Li, X.Z. Decay model solutions to cylindrical KP equation. Appl. Math. Lett. 2016, 62, 29-34. [CrossRef]

16. Wang, M.L.; Zhang, J.L.; Li, X.Z. N-dimensional auto-Backlund transformation and exact solutions to n-dimensional Burgers system. Appl. Math. Lett. 2017, 63, 46-52. [CrossRef]

17. Wang, M.L.; Li, Z.X.; Zhang, J.L. Two-soliton solution to a generalized KP equation with variable coefficients. Appl. Math. Lett. 2018, 76, 21-27. [CrossRef]

18. Abramowitz, M.; Stegun, I.A. Handbook of Mathematical Functions; Dover Publications Inc.: New York, NY, USA, 1994.

19. Zwillinger, D. Handbook of Differential Equations; Gulf Professional Publishing: Woburn, MA, USA, 1992. 